\title{
Distribution and change of peroxynitrite in the guinea pig cochlea following noise exposure
}

\author{
WEI-JU HAN ${ }^{1,2}$, XIAO-RUI SHI ${ }^{1,2}$ and ALFRED NUTTALL ${ }^{1-3}$ \\ ${ }^{1}$ Department of Otolaryngology Head and Neck Surgery, The Chinese PLA General Hospital, \\ Beijing 100853, P.R. China; ${ }^{2}$ Oregon Hearing Research Center, OHSU, Portland, OR 97239; \\ ${ }^{3}$ KHRI, University of Michigan, Ann Arbor, MI 48109-5616, USA
}

Received June 20, 2017; Accepted July 10, 2017

DOI: $10.3892 /$ br.2018.1107

\begin{abstract}
Nitric oxide (NO)-mediated pathology depends on the formation of reactive intermediates, such as the peroxynitrite $\left(\mathrm{ONOO}^{-}\right)$. $\mathrm{ONOO}^{-}$can nitrate free tyrosine and tyrosine residues of proteins. Therefore, increases in tyrosine nitration reflect the amount of $\mathrm{ONOO}^{-}$produced by oxidative stress. The distribution of 3-nitrotyrosine (3-NT), an ONOOmarker, in the organ of corti and the cochlear lateral wall tissue from the guinea pig were examined using fluorescence immunohistochemistry. The immunoactivity of 3-NT in the normal guinea pig was compared with animals exposed to $122 \mathrm{dBA}$ broadband noise, $4 \mathrm{~h} / \mathrm{day}$, for 2 consecutive days. In the normal animals, 3-NT immunoreactivity was found in the outer hair cells (OHCs), inner hair cells (IHCs), pillar cells (PCs), spiral ganglion cells (SPCs) and the marginal cells of stria vascularis in the lateral wall. Sound exposure increased the 3-NT signal in all of the cells and resulted in extensive outer hair cell loss. A quantitative analysis of the 3-NT change in OHCs and marginal cells of lateral wall showed that immunolabeling was significant $(\mathrm{P}<0.01, \mathrm{n}=10)$ in the noise exposure group compared with that of the control group. Anti-3-NT and propidium iodide double labeling showed that 3-NT was distributed mainly in the apical end of OHCs. In addition, 3-NT was distributed outside of the nucleus of the OHCs and marginal cells. In conclusion, the data indicate that noise exposure leads to a significant production of $\mathrm{ONOO}^{-}$in the cochlear lateral wall and organ of corti. This is consistent with the known increase of NO production by loud sound stress and suggests that NO-derived free radicals participate in the cochlear pathophysiology of noise-induced hearing loss.
\end{abstract}

Correspondence to: Professor Wei-Ju Han, Department of Otolaryngology Head and Neck Surgery, The Chinese PLA General Hospital, 28 Fuxing Road, Beijing 100853, P.R. China

E-mail: hanweiju@aliyun.com

Key words: noise exposure, peroxynitrite, 3-nitrotyrosine, cochlea

\section{Introduction}

The controlled production of nitric oxide (NO) has an important role in mediating neurotransmission and regulating vascular tone (1). However, early after the finding of the signal transducing physiological functions of the free radical NO in the vasculature and nervous system, it became evident that NO could also participate as a cytotoxic effector molecule and/or a pathogenic mediator when produced at high rates by either inflammatory stimuli-induced NO synthase (iNOS) or overstimulation of the constitutive forms (eNOS and nNOS). Much of NO-mediated pathogenicity depends on the formation of secondary intermediates such as peroxynitrite anion $\left(\mathrm{ONOO}^{-}\right)$ and nitrogen dioxide $\left(\mathrm{NO}_{2}\right)$ that are typically more reactive and toxic than $\mathrm{NO}(2,3)$.

$\mathrm{ONOO}^{-}$is a powerful oxidant and cytotoxic agent formed by the near-diffusion limited reaction between NO and superoxide $\left(\mathrm{O}_{2}^{-}\right)(4,5)$. ONOO- can damage DNA, membrane lipids, and mitochondria, and has been shown to modify proteins at intrinsic methionine, tryptophan, and cysteine residues (6). The most important property of $\mathrm{ONOO}^{-}$is its ability to nitrate free tyrosine and tyrosine residues in proteins (7). One of the molecular footprints left by the reactions of reactive nitrogen species with biomolecules is nitration (i.e., addition of the nitro group, $\mathrm{NO}_{2}$ ) of protein tyrosine residues to 3-nitrotyrosine (3-NT). It is generally suggested that increases in tyrosine nitration, whether tyrosine is free or part of a polypeptide chain, reflect the actions of $\mathrm{ONOO}^{-}$(8). Nitration pathways involve free radical biochemistry with carbonate radicals and/or oxo-metal complexes oxidizing tyrosine to tyrosyl radical followed by the diffusion-controlled reaction with $\mathrm{NO}_{2}$ to yield 3-NT. Although protein tyrosine nitration is a low-yield process in vivo, 3-NT has been revealed as having a higher yield and is a relevant biomarker of NO-dependent oxidative stress.

Previous studies (9) have suggested that noise exposure leads to hair cell death and significant production of nitrotyrosine in the OHCs and stria vascularis. The purpose of this study was to demonstrate distribution of 3-NT, ONOOmarker, in the organ of corti and in the lateral wall of the cochlea in the guinea pig and to examine the influence of broad band noise exposure to 3 -NT distribution in the cochlea. 


\section{Materials and methods}

Animal preparation. A total of 24 guinea pigs (albinos of both sexes, 200-250 g) with normal hearing were used in this study. The animals were divided into the control and experimental groups. Animals in the control group $(n=12)$ were kept in a quiet room with food and water (constant temperature of $21-24^{\circ} \mathrm{C}$ and humidity of 40-70\%). Animals in the experimental group $(n=12)$ were placed in the noise exposure chamber and exposed for $4 \mathrm{~h} /$ day to broadband noise at $122 \mathrm{~dB}$ SPL (A-weighted) for 2 consecutive days. Hearing of the animal was estimated using auditory brain-stem response thresholds before and after noise exposure. This exposure level resulted in a permanent threshold shift (10). Guinea pigs were processed for the detection of the distribution and change of 3-NT in the organ of corti and cochlear lateral wall immediately following noise exposure.

Specimen preparation. The animals were anesthetized and cardiac perfusion was performed with saline following the second day's noise exposure, then a further vascular perfusion with $4 \%$ paraformaldehyde followed. One ear from each animal was used for whole mount surface preparation, while the contralateral ear was used for frozen section methods. The cochlea was gently isolated after fixing. For whole mount preparation, the lateral wall tissues and the organ of corti were harvested after removal of the bony capsule. For the frozen section methods, cochleae were harvested with the bony capsule. After rinsing with $0.1 \mathrm{M} \mathrm{PB}$, the cochleae were decalcified in 8\% ethylene diamine tetraacetic acid (EDTA) in PBS in microwave-assisted decalcification. After infiltration with 10 and $30 \%$ sucrose, the cochlea was then placed overnight into a Tissue-Tek mold and frozen in the mold with dry ice. Eight-micron cryostat (Bright Instrument, Huntingdon, Cambridgeshire, UK) sections were obtained, and thaw-mounted onto slides pre-coated with silane (Sigma Diagnostics, Inc., St. Louis, MO, USA). The tissue was then processed for immunohistochemistry.

Immunohistochemistry. The specimens were incubated overnight in anti-3-NT (mouse monoclonal antibody, 39B6; Alexis Biochemicals, San Diego, CA, USA). The specimens were washed in 1\% BSA-PBS for $30 \mathrm{~min}$ and incubated in Alexa Fluor 488 anti-mouse $\operatorname{IgG}$ (catalog no. A11001) for 3-NT and Alexa Fluor 568 phalloidin (diluted 1:50 with 1\% BSA-PBS; Molecular Probes Life Technologies, Carlsbad, CA, USA) for $1 \mathrm{~h}$. After washing in 0.02 PBS for $30 \mathrm{~min}$, the surfaceprepared tissues and frozen sections were mounted separately. Negative controls were incubated tissue with $1 \%$ BSA-PBS to replace the primary antibody. In addition to immunoactivity labeling, the specimens were double labeled either with Alexa Fluor 568 phalloidin, a probe for F-actin, to observe the whole cell shape or with propidium iodide, a DNA intercalator, to observe nuclear morphology.

Fluorescence and confocal microscope. The laser scanning confocal microscope (MRC 1024ES; Bio-Rad, Berkeley, CA, USA) was used to observe the fluorescent signals. Images were captured using the same settings for gain and illumination power. Cochleae were double labeled with red fluorescence
(PI-labeled nuclei and phalloidin labeled F-actin) and green fluorescence (Alexa Fluor 488 anti-rabbit antibody labeled 3-nitrotyrosin). A series of 40-60 laser confocal images were taken for each section of the organ of corti beginning at the top of the $\mathrm{OHC}$ stereocilia and stepping progressively through the OHCs body to the bottom of the OHCs at depth intervals of $1.0 \mu \mathrm{m}$. The images in the figures are presented as projection sets of 40-60 images.

Statistical analysis. Quantitative analysis for fluorescence intensity of 3-NT was performed using Adobe Photoshop 7.0 software on single confocal optical sections. To quantitate 3-NT in OHCs and marginal cells of cochlear lateral wall before and after noise exposure, the image to be analyzed was chosen as the brightest $\mathrm{Z}$-axis confocal section at the level of the OHCs or marginal cells. The mean fluorescent intensity was determined using the software over a user-selected window approximately covering the organ of corti or marginal cells in the acquired image. A mean background fluorescence level was determined in a small window located away from the fluorescence tissue, and was subtracted from the mean fluorescence intensity value. The data were averaged within the 10 experimental and 10 control animals. All values presented in Results are mean \pm SD. Differences among the different groups were evaluated using a two-tailed Student's t-test. $\mathrm{P}<0.05$ was considered to indicate a statistically significant difference.

\section{Results}

Distribution and change of $\mathrm{ONOO}^{-}$in the normal and noise-stimulated organ of corti. The distribution of 3-NT,

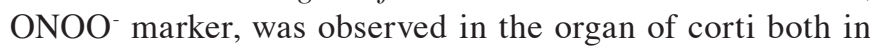
the control and experimental groups. For the normal organ of corti, relatively weak 3-NT immunolabeling was detected in the OHCs, pillar cells (PCs), inner hair cells (IHCs), and spiral ganglion cells (SPCs) (Fig. 1A and C). After animals were exposed for $4 \mathrm{~h} / \mathrm{day}$ to broadband noise at $122 \mathrm{~dB}$ SPL (A-weighted) for two consecutive days, the activity of 3-NT immunostaining became stronger immediately following the second day's noise exposure in various cochlear cells. The increased labeling was especially prominent in OHCs, PCs and SPCs (Fig. 1D and F). The same phalloidin staining was observed in control (panel B) and noise exposure animal (panel E): However, quite a few OHCs were lost in the noise exposure animal (panels D-F). No hair cell loss was observed in the control animal (panels A-C).

Noise induced $\mathrm{ONOO}^{-}$increase in $\mathrm{OHCs}$ from guinea pig cochlea. The significant change of 3-NT, ONOO- marker, was observed in OHCs. To quantitatively determine 3-NT increase in OHCs following noise exposure, the mean fluorescent intensity was measured using the software in a user-selected window approximately covering the OHCs images in the 10 control animals and in the 10 noise exposure animals. We found weak 3-NT signals in the OHCs of control animals (Fig. 2A). The stronger 3-NT signals were observed in OHCs immediately following two days of noise exposure (Fig. 2B). There was no apparent immunoreactivity in OHCs when incubated with the secondary antibody alone (Fig. 2C). The quantitative analysis 

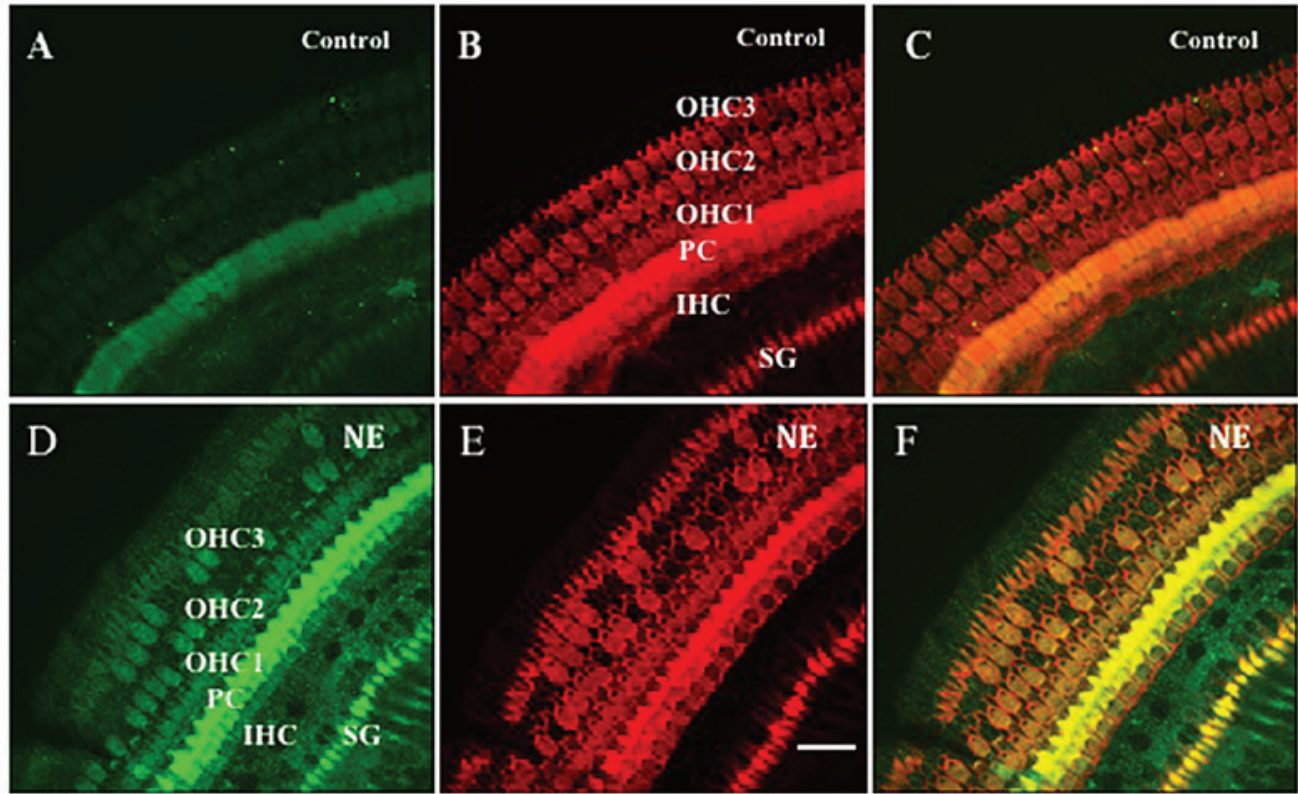

Figure 1. Anti-3-NT and phalloidin double staining showed the 3-NT distribution in the organ of corti before and after NE. (A-C) Control group; (D-F) NE group. The organs of corti were stained (red, B and E) with phalloidin showing whole cell shape and (green, A and D) anti-3-NT antibody showing the distribution of 3-NT. Panel C is the merged image of A and B and panel F is that of D and E. Relatively weak staining of 3-NT was found in the OHCs, IHCs, SPCs and PCs in the control animal (panels A and C). Significant 3-NT immunostaining increase was observed in the OHCs, IHCs, PCs and SPCs in the NE animal (panels D and F). The same phalloidin staining was observed in control (panel B) and noise exposure animals (panel E); however, quite a few OHCs were lost in the noise exposure animal (panels D-F). No hair cell loss was observed in the control animal (panels A-C). 3-NT, anti 3-NT; OHC 1-3: first, second and the third row of OHCs; PC, pillar cell; SG, spiral ganglion. Scale bar, $20 \mu \mathrm{m}$. 3-NT, 3-nitrotyrosine; NE, noise exposure; OHCs, outer hair cells; IHCs, inner hair cells; SPCs, spiral ganglion cells; PCs, pillar cells.
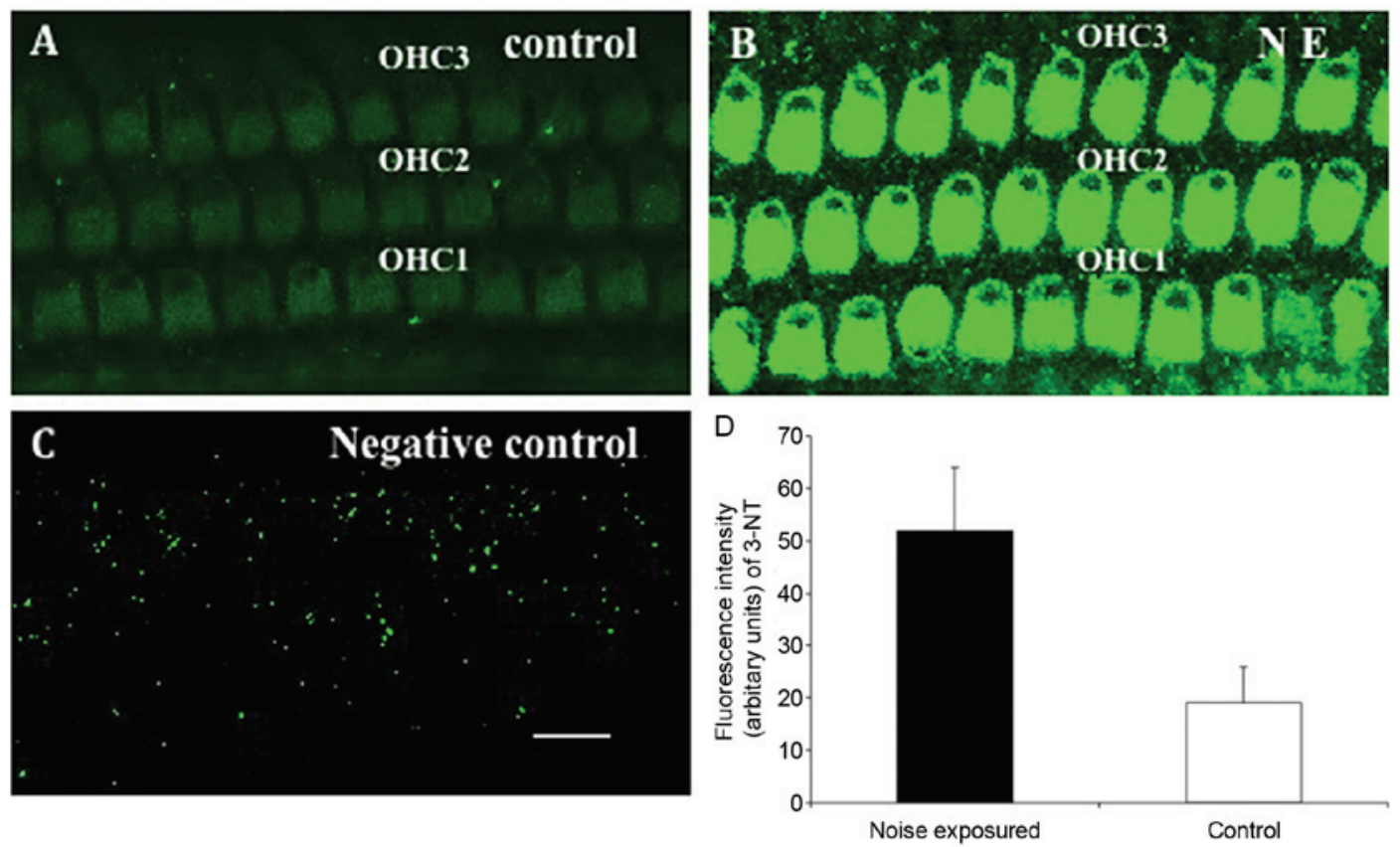

Figure 2. 3-NT increase in OHCs following noise exposure. (A) Immunostaining of anti-3-NT in the organ of corti of the control animal showed a relatively weak staining of 3-NT in the OHCs. (B) Significant 3-NT immunostaining increase was observed in the OHCs of guinea pig cochleae following NE. (C) No immunoreactive staining was observed in the control animals without the primary antibody. (D) A histogram comparing the intensity of 3-NT fluorescence in the control and noise-exposed group $(\mathrm{P}<0.01, \mathrm{n}=10)$. 3-NT, anti 3-NT; OHC 1-3: first, second and third row of OHCs. Scale bar, $10 \mu \mathrm{m}$. 3-NT, 3-nitrotyrosine; $\mathrm{NE}$, noise exposure; OHCs, outer hair cells.

showed 3-NT signals of OHCs significantly increased $(\mathrm{P}<0.01$, $\mathrm{n}=10$ ) in the noise exposure group compared with that of the control group.
ONOO distribution outside of nuclei in OHCs. To further determine 3-NT distribution inside of OHCs, the organs of corti were double labeled with anti-3-NT, to show 3-NT 

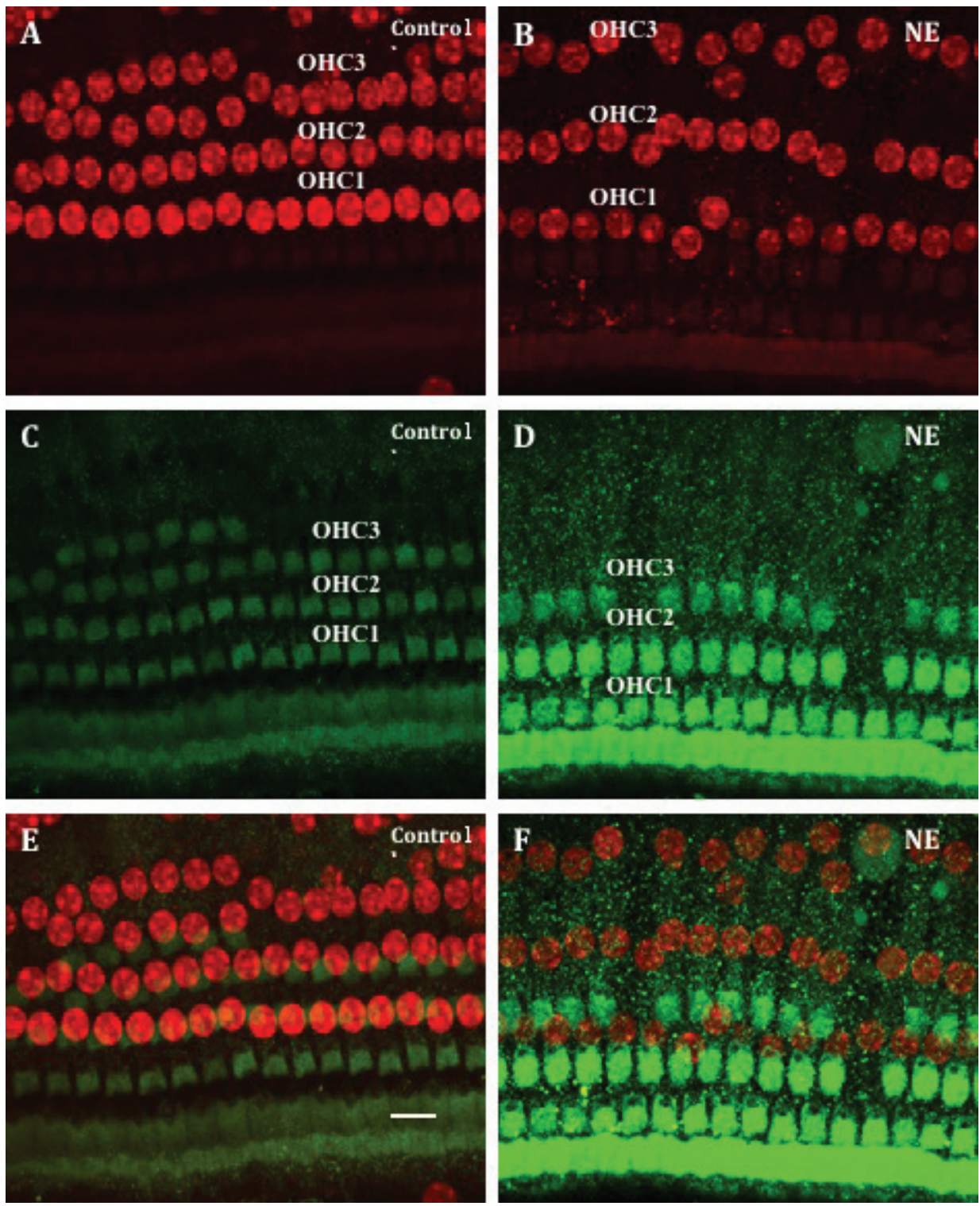

Figure 3. Anti-3-NT and propidium iodide double staining showed the 3-NT distribution in the hair cells before and after noise exposure. (A, C and E) Control. $(\mathrm{B}, \mathrm{D}$ and F) NE. The hair cells were stained (red, A and B) with propidium iodide showing nucleoli and (green, $\mathrm{C}$ and D) anti-3-NT antibody showing the distribution of 3-NT. Panel E is the merged image of A and C and panel F is that of B and D. Relatively weak staining of 3-NT was found in the apical end of OHCs in the control animals (panels C and E). Significant 3-NT immunostaining increase was observed in the apical end of OHCs in the noise-exposed animals (panels D and F). The same propidium iodide staining was observed in control (panel A) and NE animals (panel B). Anti-3-NT and propidium iodide double staining showed that 3-NT is distributed mainly in the apical end of hair cells and no distribution is seen in the nuclear area (panels E and F). OHC 1-3: first, second and third row of OHCs. Scale bar, $20 \mu \mathrm{m}$. 3-NT, 3-nitrotyrosine; NE, noise exposure; OHCs, outer hair cells.

distribution, and propidium iodide, to show nuclei in both the control and noise exposure groups. Relatively weak staining of 3-NT was found in the apical end of OHCs in the control animals (Fig. 3C and E). A significant 3-NT immunostaining increase was observed in the apical end of $\mathrm{OHCs}$ in the noise exposure animals (Fig. 3D and F). Anti-3-NT and propidium iodide double staining showed that 3-NT is distributed mainly in the apical end of hair cells and no distribution was evident in the nuclear area (panels $\mathrm{E}$ and F).

$\mathrm{ONOO}^{-}$distribution in the cochlear lateral wall in the normal and noise exposure guinea pig cochleae. The distributions of 3-NT, ONOO- marker, were observed in cryostat sections of the cochlear lateral wall. The frozen sections were double labeled with anti-3-NT and propidium iodide. In the unstimulated guinea pig lateral wall, relatively weak 3-NT immunolabeling was observed in stria vascularis (Fig. 4A and B). The 3-NT immunoactivity became stronger in stria vascularis in the tissue specimens from noise-exposed animals (Fig. 4C and D). There was no apparent immunoreactivity in the spiral ligament area of the cochlear lateral wall. Anti-3-NT and propidium iodide double staining showed that 3-NT distributes mainly in the outside of the nucleus of the marginal cells.

Noise exposure-induced $\mathrm{ONOO}^{-}$increase in the marginal cells from the lateral wall of guinea pig cochleae. ONOOdistributions in cochlear lateral wall were also observed in surface-prepared specimens from the control and noise-exposed animals. 3-NT immunoactivity was quantitatively analyzed in the marginal cells of lateral wall. Relatively weak staining 

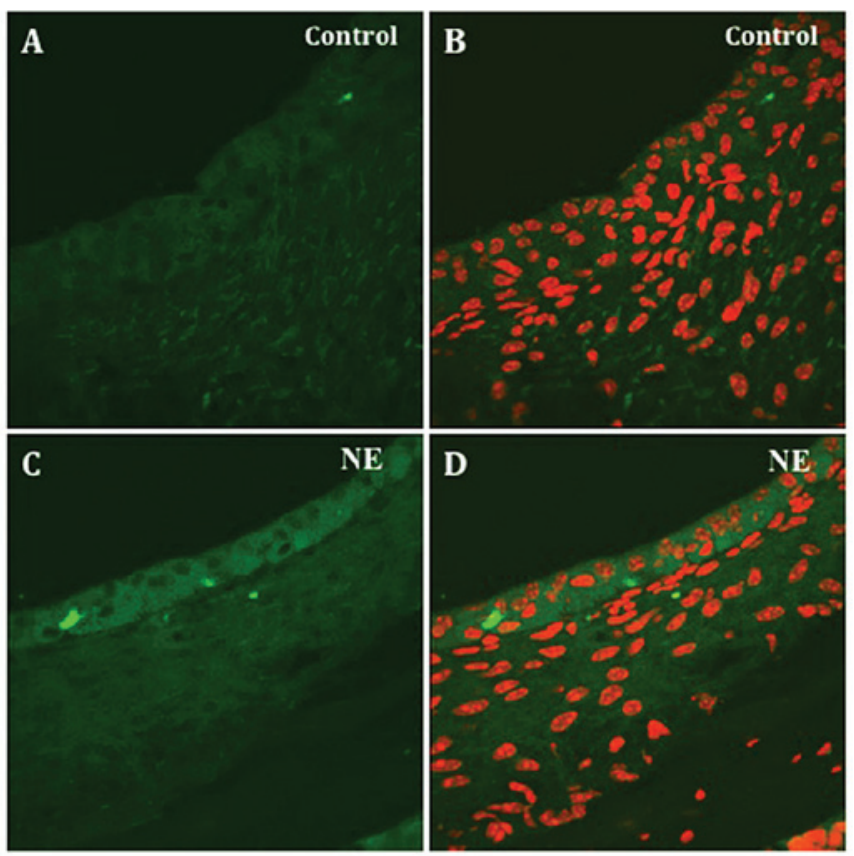

Figure 4. Anti-3-NT and propidium iodide double staining in the frozen sections in the cochlear lateral wall of the control and NE guinea pig. (Panels A and B) Relatively weak staining of 3-NT was found in the stria vascularis in the control animal. By contrast, enhanced 3-NT immunostaining was observed (panels $\mathrm{C}$ and $\mathrm{D}$ ) in the stria vascularis in the $\mathrm{NE}$ animal. Anti-3-NT and propidium iodide double staining showed that 3-NT distribute mainly in the outside of the nucleus of the marginal cells. 3-NT, 3-nitrotyrosine; NE, noise exposure.

of 3-NT was found in marginal cells in the control animal (Fig. 5A). Significant 3-NT immunostaining increase was observed in the marginal cells in the noise-exposed animal (Fig. 5D and G). The same phalloidin staining was observed in the control (Fig. 5D) and noise-exposed animals (Fig. 5E). Anti-3-NT and propidium iodide double staining showed that 3-NT distributes mainly outside of nuclei in the marginal cells and no distribution is seen in the nuclear area (Fig. 5H and I). Quantitative analysis comparing the intensity of 3-NT fluorescence in the control and noise-exposed groups showed a significant $(\mathrm{P}<0.01, \mathrm{n}=10) 3-\mathrm{NT}$ increase in the marginal cells (Fig. 5J) following noise exposure.

\section{Discussion}

Previous findings have shown that NO may play an important role in the regulation of cochlear blood flow and auditory neurotransmission $(11,12)$. Much of NO-mediated pathogenicity would depend on the formation of secondary intermediates. $\mathrm{NO}$ readily diffuses through the cytosol and cell membrane, allowing it to react with $\mathrm{O}_{2}^{-}$to form $\mathrm{ONOO}^{-}$. Thus, the increase in tyrosine nitration reflects the actions of $\mathrm{ONOO}^{-}$. 3-NT can be a marker for $\mathrm{ONOO}^{-}$-induced neurotoxicity (13). ONOO that are typically more reactive and toxic than NO.

In the present study, we have examined the distribution of NO derivatives, 3-NT, in the cochlear lateral wall tissue and the organ of corti from the guinea pig using fluorescence immunohistochemistry. We found that in the normal (no noise exposure) animals, 3-NT was observed in the OHCs, IHCs,
PCs, SPCs and the marginal cells of stria vascularis in the lateral wall. This result is consistent with a previous report which identified the presence of NO in the IHCs, OHCs, and the stria vascularis (14). Although the complete actions of $\mathrm{ONOO}^{-}$remain to be determined, we clearly found 3-NT signals in OHCs and IHCs as well as PCs and SPCs. 3-NT distributions reflect the diffusion of NO, products of NADPH oxidase in mitochondria and the location of tyrosine. Since $\mathrm{NO}$ is a small molecule and a gas that freely diffuses (but is short-lived), it is difficult to determine the precise location of its production. Thus, our data of 3-NT distributions mainly reflect the location of $\mathrm{O}_{2}^{-}$producing and tyrosine nitration. Mitochondria have been recognized as critical sources and targets of nitrating species (15). Although the formation of NO mainly occurs outside the mitochondria, the diffusion of NO into mitochondria and its combination with mitochondrial-derived $\mathrm{O}_{2}^{-}$results in the formation of $\mathrm{ONOO}^{-}$, which accounts for much of the disruption of mitochondrial metabolism initially attributed to direct actions of NO and to the nitration of mitochondrial proteins. In the present study, anti-3-NT and propidium iodide double staining showed that 3-NT distributes mainly in the apical end of OHCs, and 3-NT distributes outside of the nucleus of the OHCs. This result is consistent with mitochondria as sources and targets of nitrating species.

Noise-induced hearing loss reflects a direct mechanical trauma occurring during noise exposure and oxidative stress-induced formation of reactive oxygen species (ROS). ROS are free radicals, reactive molecules containing oxygen, or molecules containing oxygen that generate free radicals. ROS include $\mathrm{NO}, \mathrm{O}_{2}^{-}, \mathrm{ONOO}^{-}$and hydrocynical radical. These reactive species directly destroy DNA and cell membranes, and act as signaling molecules for the upregulation of apoptotic cell death genes. Thus, hydroxyl radicals significantly increase in the cochlea with noise, nitrotyrosine and 4-hydroxy-2-nonel increase within 10 days following noise exposure (16), isoprostanes, directly reflecting ROS formation after intense noise, form in the organ of corti and lateral wall, and oxidative-induced DNA damage follows intense noise exposure (9). In the present study, we quantitatively analyzed the change of 3-NT in OHCs before and after noise exposure. We found a significant increase of $3-\mathrm{NT}$ in $\mathrm{OHCs}(\mathrm{P}<0.01$, $\mathrm{n}=10$ ) in the noise exposure group compared with that of the control group. The increase of 3-NT means large quantities of $\mathrm{ONOO}^{-}$production under loud sound stimulation. This excess $\mathrm{ONOO}^{-}$is the principal pathogenic pathway resulting from the reaction of $\mathrm{NO}$ with oxygen and oxygen radicals. The increased 3-NT means the formation of reactive NO species, which are thought to play a crucial role in cell injury and death by a complex process, including damage to DNA and mitochondria, resulting in the disruption of energy metabolism (17). Using phalloidin labeling, we observed quite a few hair cell losses following loud sound stimulation. In addition, apoptosis and necrosis were observed in OHCs after noise exposure (17). Some studies have suggested that mitochondria contain NO synthase and are capable of producing biologically significant quantities of NO to regulate energy metabolism and perform other physiologic functions or to become involved in pathologic processes. NO-related free radicals can damage the organelles leading to cell death through irreversible inhibition 

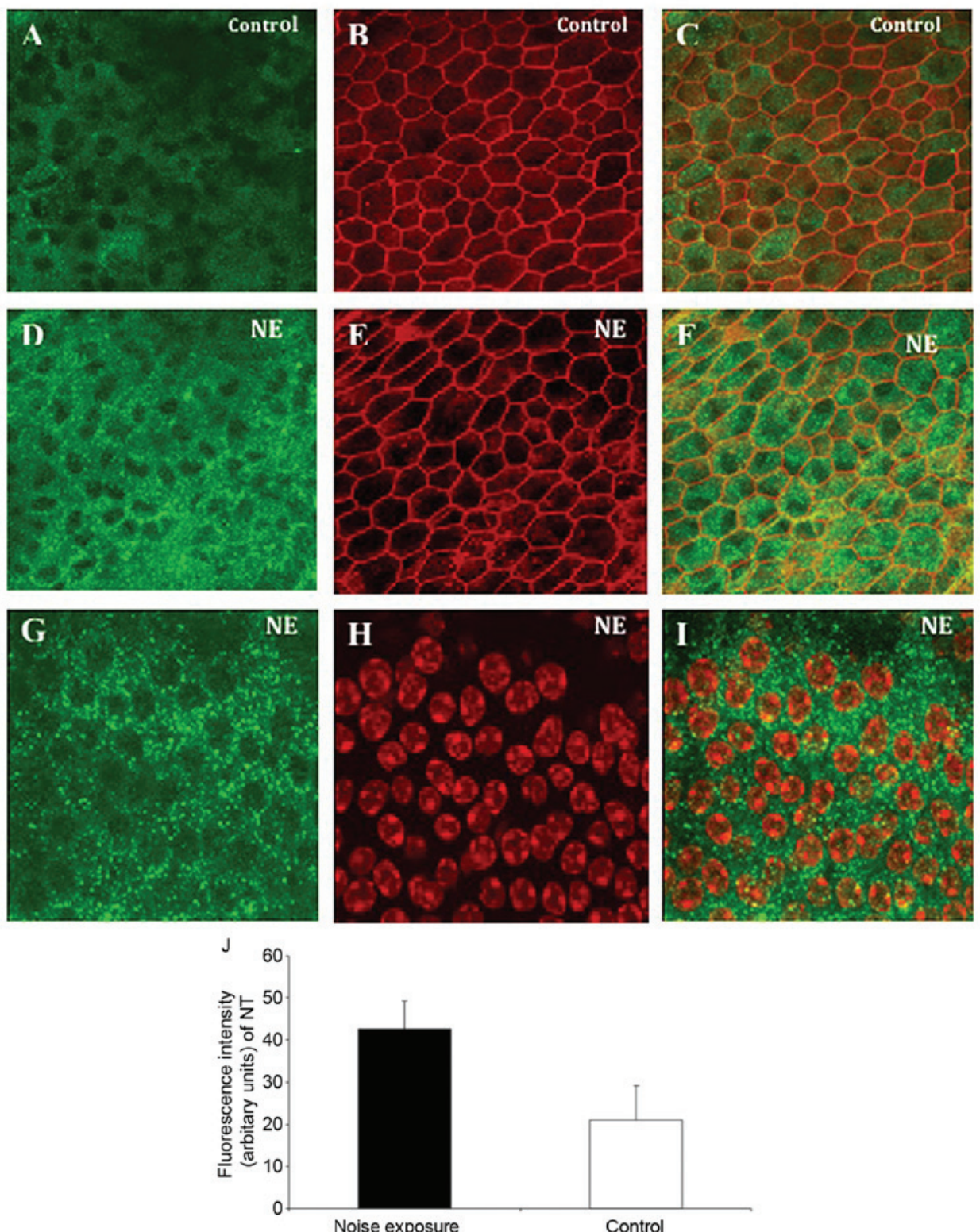

Figure 5. Anti-3-NT and propidium iodide (or phalloidin) double staining showed the 3-NT distribution in the marginal cells of cochlear lateral wall before and after noise exposure. (A, B and C) Control group; (D-H and J) NE group. The marginal cells were stained with propidium iodide showing nuclei (H), phalloidin showing whole cell shape (B and E) and anti-3-NT antibody showing the distribution of 3-NT (green, A, D and G). Panel C is the merged image of A and B, panel $\mathrm{F}$ is that of D and E, and panel I is that of $\mathrm{G}$ and $\mathrm{H}$. Relatively weak staining of 3-NT was found in marginal cells in the control animals (panel A). Significant 3-NT immunostaining increase was observed in the marginal cells in the noise-exposed animals (panels D and G). The same phalloidin staining was observed in the control (panel B) and NE animals (panel E). Anti-3-NT and propidium iodide double staining showed that 3-NT distributes mainly out of nuclei in the marginal cells and no distribution is seen in th nuclear area (panels H and I). A histogram (panel J) comparing the intensity of 3-NT fluorescence in the control and NE groups showed the significant $(\mathrm{P}<0.01, \mathrm{n}=10)$ 3-NT increase in the marginal cells of the cochlear lateral wall. 3-NT, 3-nitrotyrosine; NE, noise exposure.

of mitochondrial respiration or other damage to a variety of mitochondrial components via oxidizing reactions involving ONOO- $^{-}(18,19)$.

In the present study, both whole mount and cryostat sections were performed to observe the distribution of 3-NT in the marginal cells of stria vascularis in the lateral wall. We observed relatively weak 3-NT distributions in marginal cells in unstimulated animals (Figs. 4A and 5A). By contrast, upregulated 3-NT immunoactivity was detected in the marginal cells following exposure to the broadband noise. The quantitative analysis revealed $3-\mathrm{NT}$ in marginal cells of lateral wall increased significantly $(\mathrm{P}<0.01, \mathrm{n}=10)$ in the noise exposure group compared with that of the control group. This is consistent with previous report that increased NO signal was observed following exposure to noise (14). A high level of $\mathrm{NO}$ can react rapidly with $\mathrm{O}_{2}^{-}$to produce $\mathrm{ONOO}^{-}$, which are strong oxidants that could produce marginal cell damage. An early study showed that $\mathrm{O}_{2}{ }^{-}$anion radicals emerged along the luminal membrane of the marginal cells of the SV following acoustic trauma in the guinea pig (20). We have observed increased 3-NT activity in marginal cells, which may result in oxidative injury to those cells due to $\mathrm{ONOO}^{-}$related free radical damage, and may cause loss of homeostasis of the endolymph. 
Taken together, this study demonstrates the presence of 3-NT, an $\mathrm{ONOO}^{-}$marker, in the IHCs, OHCs, PCs, SPCs and the marginal cells of stria vascularis in the normal guinea pig cochlea. The 3-NT distributes mainly in the apical end of hair cells and no distribution is seen in the nuclear area. After the noise exposure, the increased 3-NT signals were observed in the IHCs, OHCs, PCs, SPCs and marginal cells of the stria vascularis. Furthermore, to increase nitrotyrosine and 3-NT, hair cell loss was observed for the $\mathrm{OHCs}$ following noise exposure.

\section{Acknowledgements}

The present study was supported by grants from National Natural Science Foundation of China (nos. 81470683 and 81770992).

\section{References}

1. Michel O, Hess A, Bloch W, Stennert E, Su J and Addicks K Localization of the NO/cGMP-pathway in the cochlea of guinea pigs. Hear Res 133: 1-9, 1999.

2. Radi R: Nitric oxide, oxidants, and protein tyrosine nitration. Proc Natl Acad Sci USA 101: 4003-4008, 2004.

3. Gheddouchi S, Mokhtari-Soulimane N, Merzouk H, Bekhti F, Soulimane F, Guermouche B, Meziane Tani A and Narce M: Low SOD activity is associated with overproduction of peroxynitrite and nitric oxide in patients with acute coronary syndrome. Nitric Oxide 49: 40-46, 2015.

4. Koppenol WH, Moreno JJ, Pryor WA, Ischiropoulos H and Beckman JS: Peroxynitrite, a cloaked oxidant formed by nitric oxide and superoxide. Chem Res Toxicol 5: 834-842, 1992.

5. Huie RE and Padmaja S: The reaction of no with superoxide. Free Radic Res Commun 18: 195-199, 1993.

6. Beckman JS and Koppenol WH: Nitric oxide, superoxide, and peroxynitrite: The good, the bad, and ugly. Am J Physiol 271: C1424-C1437, 1996.

7. Ischiropoulos $\mathrm{H}$ : Biological selectivity and functional aspects of protein tyrosine nitration. Biochem Biophys Res Commun 305: 776-783, 2003.

8. Crow JP and Ischiropoulos H: Detection and quantitation of nitrotyrosine residues in proteins: In vivo marker of peroxynitrite. Methods Enzymol 269: 185-194, 1996.
9. Han WJ, Shi XR and Nuttall A: Noise-induced nitrotyrosine increase and outer hair cell death in guinea pig cochlea. Chin Med J (Engl) 126: 2923-2927, 2013.

10. Zhang J, Chen X, Wu J and Han W: Detection of guinea pigs hearing functions before and after white noise exposure. Chin J Otol 31: 141-144, 2015.

11. Konishi K, Yamane H, Iguchi H, Takayama M, Nakagawa T, Sunami K and Nakai Y: Local substances regulating cochlear blood flow. Acta Otolaryngol Suppl 538: 40-46, 1998.

12. Hess A, Bloch W, Huverstuhl J, Su J, Stennert E, Addicks K and Michel O: Expression of inducible nitric oxide synthase (iNOS/NOS II) in the cochlea of guinea pigs after intratympanical endotoxin-treatment. Brain Res 830: 113-122, 1999a.

13. Kuhn DM, Sakowski SA, Sadidi M and Geddes TJ: Nitrotyrosine as a marker for peroxynitrite-induced neurotoxicity: The beginning or the end of the end of dopamine neurons? J Neurochem 89: 529-536, 2004.

14. Shi X, Ren T and Nuttall AL: Nitric oxide distribution and production in the guinea pig cochlea. Hear Res 153: 23-31, 2001.

15. Radi R, Denicola A, Alvarez B, Ferrer-Sueta G and Rubbo H: Nitric Oxide. Ignarro L (ed). Academic Press, San Diego, pp57-82, 2000.

16. Yamashita D, Jiang HY, Schacht J and Miller JM: Delayed production of free radicals following noise exposure. Brain Res 1019: 201-209, 2004.

17. Van Campen LE, Murphy WJ, Franks JR, Mathias PI and Toraason MA: Oxidative DNA damage is associated with intense noise exposure in the rat. Hear Res 164: 29-38, 2002.

18. Brown GC: Nitric oxide as a competitive inhibitor of oxygen consumption in the mitochondrial respiratory chain. Acta Physiol Scand 168: 667-674, 2000.

19. Pisoschi AM and Pop A: The role of antioxidants in the chemistry of oxidative stress: A review. Eur J Med Chem 97: 55-74, 2015.

20. Yamane H, Nakai Y, Takayama M, Konishi K, Iguchi H, Nakagawa T, Shibata S, Kato A, Sunami K and Kawakatsu C: The emergence of free radicals after acoustic trauma and strial blood flow. Acta Otolaryngol Suppl 519: 87-92, 1995.

This work is licensed under a Creative Commons Attribution-NonCommercial-NoDerivatives 4.0 International (CC BY-NC-ND 4.0) License. 